\title{
Cost-effectiveness of adding novel or group 5 interventions to a background regimen for the treatment of multidrug-resistant tuberculosis in Germany
}

\author{
Daniel Wirth ${ }^{1 *}$, Ramesh Dass $^{2}$ and Robert Hettle ${ }^{3}$
}

\begin{abstract}
Background: Treatment of multidrug-resistant tuberculosis (MDR-TB) is complex, lengthy, and involves a minimum of four drugs termed a background regimen (BR), that have not previously been prescribed or that have proven susceptible to patient sputum culture isolates. In recent years, promising new treatment options have emerged as add-on therapies to a BR. The aim of this study was to evaluate the long-term costs and effectiveness of adding the novel or group 5 interventions bedaquiline, delamanid, and linezolid to a background regimen (BR) of drugs for the treatment of adult patients with pulmonary multidrug-resistant tuberculosis (MDR-TB), within their marketing authorisations, from a German healthcare cost-effectiveness perspective.
\end{abstract}

Methods: A cohort-based Markov model was developed to simulate the incremental cost-effectiveness ratio of bedaquiline plus BR, delamanid plus BR, or linezolid plus BR versus BR alone in the treatment of MDR-TB, over a 10-year time horizon. Effectiveness of treatment was evaluated in Quality-Adjusted Life-Years (QALYs) and LifeYears Gained (LYG), using inputs from clinical trials for bedaquiline and delamanid and from a German observational study for linezolid. Cost data were obtained from German Drug Directory costs (€/2015), published literature, and expert opinion. A 3\% yearly discount rate was applied. Probabilistic and deterministic sensitivity analyses were conducted.

Results: The total discounted costs per-patient were $€ 85,575$ for bedaquiline plus BR, $€ 81,079$ for delamanid plus BR, and $€ 80,460$ for linezolid plus BR, compared with a cost of $€ 60,962$ for BR alone. The total discounted QALYs per-patient were 5.95 for bedaquiline plus BR, 5.36 for delamanid plus BR, and 3.91 for linezolid plus BR, compared with 3.68 for BR alone. All interventions were therefore associated with higher QALYs and higher costs than BR alone, with incremental costs per QALY gained of $€ 22,238$ for bedaquiline, $€ 38,703$ for delamanid, and $€ 87,484$ for linezolid, versus BR alone. In a fully incremental analysis, bedaquiline plus BR was the most cost-effective treatment option at thresholds greater than $€ 22,000$ per QALY gained. In probabilistic analyses, the probability that bedaquiline plus BR was the most cost-effective treatment strategy at a willingness-to-pay threshold of $€ 30,000$ was $54.5 \%$, compared with $22.9 \%$ for BR alone, $18.2 \%$ for delamanid plus BR, and $4.4 \%$ for linezolid.

Conclusions: In Germany, the addition of bedaquiline, delamanid, or linezolid to a BR would result in QALY gains over $\mathrm{BR}$ alone. Based on this analysis, bedaquiline is likely to be the most cost-effective intervention for the treatment of MDRTB, when added to a BR regimen at thresholds greater than $€ 22,000$ per QALY.

Keywords: Cost-effectiveness, Multidrug-resistant tuberculosis, Bedaquiline, Delamanid, Linezolid, QALY, LYG, ICER

\footnotetext{
*Correspondence: dwirth2@its.jnj.com

${ }^{1}$ Health Economics \& Market Access, Janssen-Cilag GmbH, Johnson \&

Johnson Platz 1, 41470 Neuss, Germany

Full list of author information is available at the end of the article
} 


\section{Background}

Multidrug-resistant tuberculosis (MDR-TB) is a form of tuberculosis (TB) that is resistant to at least the two most effective first-line therapeutic drugs, isoniazid and rifampicin [1]. MDR-TB is a persistent, and in some regions, increasing public health concern: the incidence of MDR-TB in Europe in 2013 was 16.9\% among new TB cases and $48.0 \%$ among previously treated cases [2].

In Germany, the total number of MDR-TB cases has increased year-on-year between 2010 and 2013, reaching 100 to 102 reported cases in 2013, depending on the reporting method used [2,3]. Further, three cases of extensively drug-resistant (XDR)-TB in Germany were reported in 2013 [3]. These statistics reflect a trend towards increasing antibiotic resistance that is mirrored throughout many regions of Europe, both in tuberculosis and in other infectious diseases [4].

Treatment of MDR-TB is complex and involves a minimum of four drugs that have not previously been prescribed or that have proven susceptible to patient isolates (termed a background regimen, or BR). The recommended total duration of treatment is $18-24$ months and patient isolation is recommended until sputum culture conversion is achieved [5-8].

Despite these lengthy and resource-intensive regimens, success rates are suboptimal; the European Centre for Disease Control (ECDC) estimated that just 38\% of patients who started MDR-TB treatment in 2011 were cured 24 months later. 235 of 1386 patients starting MDR-TB treatment died, representing a $17 \%$ mortality rate [2, 9]. For Germany, the Robert Koch Institute published very recent data with similar treatment success rates (34\%) [10]. Further, many MDR-TB therapies are highly toxic and are associated with side effects including nausea, vomiting, peripheral neuropathy, nephrotoxicity, haemotoxicity, and ototoxicity. These side effects contribute to an additional treatment and monitoring burden, negatively affect patient quality of life, and decrease patient adherence, therefore affecting the probability of treatment success [6]. All of these factors contribute to the high economic burden of MDR-TB; a recent study estimated that the direct costs (in 2012€) of MDR-TB treatment in Germany totalled $€ 64,429$ per patient, with a further $€ 17,722$ to $€ 44,304$ in lost productivity costs [11].

It is therefore necessary to introduce more effective MDR-TB treatment regimens that lead to improved patient outcomes and reduced disease transmission, while also demonstrating a manageable adverse event (AE) profile. Recently, three promising treatment options have emerged as add-on therapies to a BR: bedaquiline, linezolid, and delamanid. These are novel or Group 5 interventions that have been shown to improve efficacy outcomes in patients with MDR-TB over BR alone [12-14].
Bedaquiline and delamanid are novel interventions, while linezolid has been repurposed as an off-label treatment option in MDR-TB.

However, new healthcare interventions must be evaluated in the context of fixed healthcare budgets. In order to effectively allocate limited healthcare resources, it is important to establish not only the efficacy, but also the cost-effectiveness of new interventions for MDR-TB. Bedaquiline plus BR has previously been demonstrated to be cost-effective versus BR alone in a range of lowincome, middle-income, and high-income settings including Germany [15-17]. Delamanid has also been shown to be cost-effective versus BR in the German setting [18]. However, no published study to date has evaluated the cost-effectiveness of these three interventions within the same model structure, or evaluated the cost implications of associated AEs.

The aim of this study was to evaluate the costeffectiveness of adding bedaquiline, delamanid, or linezolid to a BR of drugs in the German setting, including the economic impact of $\mathrm{AE}$ management for each intervention.

\section{Methods \\ Model overview}

A cohort-based Markov state transition model was developed to evaluate the long-term costs and effectiveness of adding bedaquiline, delamanid, or linezolid to a BR, compared with BR alone in the treatment of adult patients with pulmonary MDR-TB. The model was originally developed for the UK healthcare system [17], and subsequently adjusted to the perspective of the German Statutory Health Insurance system [16].

A cohort of 100 patients with MDR-TB was included in the model simulation, reflecting current epidemiological data for Germany at the time of model design [2]. Of these patients, $87.7 \%$ were assumed to be treated in the inpatient setting, over a mean duration of 89.1 days [11].

Outcomes considered in the model included direct costs, quality-adjusted life-years (QALY), life-years gained (LYG), and incremental cost-effectiveness ratio (ICER). Both costs and effectiveness were discounted in the base case at an annual rate of 3\% following local guidelines [19]. A 1-month cycle length and half-cycle correction were applied to estimate costs and outcomes, with a 10 year time horizon.

\section{Model structure}

The model structure comprised six health states: active MDR-TB, sputum culture converted MDR-TB, treatment completion (representing cure), surgery, lost to follow-up (permanent treatment failure), and death (Fig. 1) [17]. The goal of drug treatment was to induce and maintain sputum culture conversion until treatment 


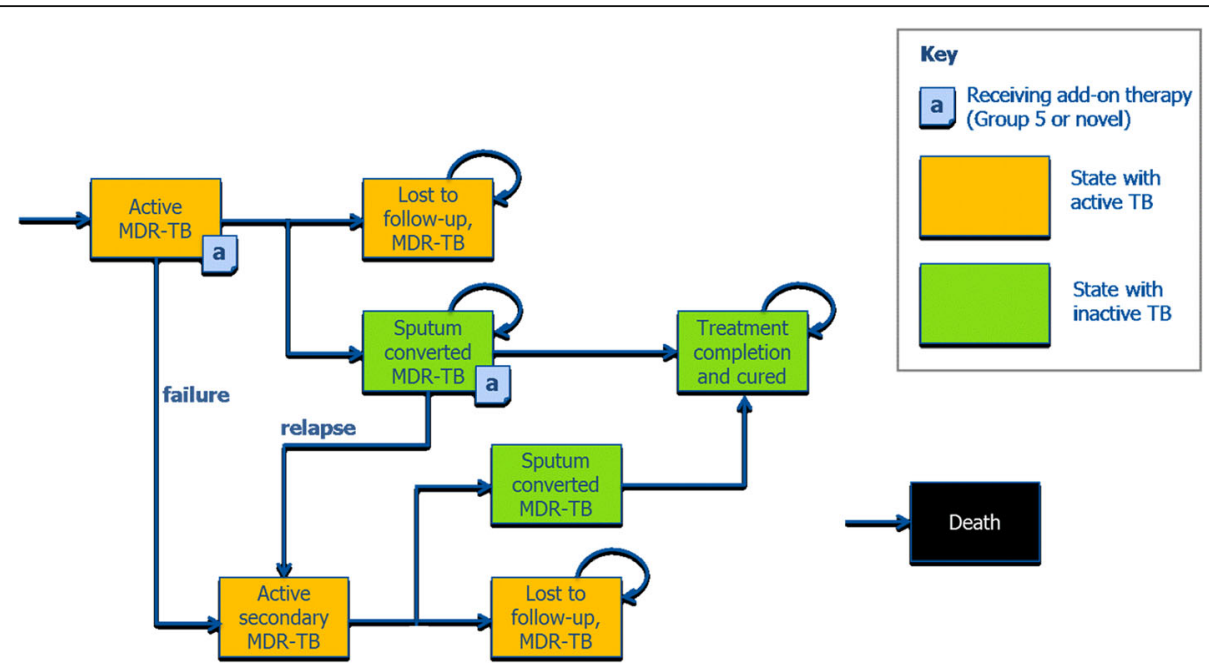

Fig. 1 Model structure, adopted from $[16,17]$. Transitions to the "Death" state are possible from every state, but not shown on the diagram for better clarity. MDR-TB: Multidrug resistant tuberculosis; TB: tuberculosis

completion (assumed equivalent to MDR-TB cure) [20]. Patients failing to achieve sputum culture conversion during the first year of the simulation were considered treatment failures and transitioned to the 'active secondary MDR-TB' state at month 12 to begin a new treatment course. Patients who failed to achieve culture conversion following the new treatment course were assumed to occupy the 'active secondary MDR-TB' state until death or loss to follow-up, for the purpose of model simplification.

The probabilities of intervention, loss to follow-up, and death were based on the literature [14, 21-24]. A summary of the transition probabilities applied in the model is shown in Table 1.

\section{Efficacy and quality of life inputs}

Efficacy data for the interventions under consideration were sourced from the results of Phase 2, placebocontrolled clinical trials for bedaquiline [14] and delamanid [12]. In both studies, the effect of treatment was evaluated in terms of the hazard ratio for time to sputum culture conversion (SCC), with bedaquiline and delamanid added to BR being associated with statistically significant improvements in the rate of SCC versus BR alone.

The rate of SCC in the population treated with add-on therapies was calculated by multiplying the hazard rate of culture conversion in the BR alone population by the hazard ratio of sputum culture conversion for treatment

Table 1 Transition probabilities in the model

\begin{tabular}{|c|c|c|c|c|}
\hline Health state & Transition to & Monthly probability (\%) & $\begin{array}{l}\text { Distribution for } \\
\text { probabilistic analysis }\end{array}$ & Source \\
\hline \multirow[t]{3}{*}{$\begin{array}{l}\text { Active TB } \\
\text { (MDR-TB; secondary MDR-TB and XDR-TB) }\end{array}$} & Sputum culture converted & Variable & $\begin{array}{l}\text { Multivariate log-normal } \\
\text { distribution }\end{array}$ & [14] \\
\hline & Lost to follow-up & 0.39 & Beta & [14] \\
\hline & Death & $\begin{array}{l}\text { MDR-TB, no cure: } 2.21, \text { MDR-TB, } \\
\text { cured: } 0.32 \\
\text { XDR-TB, no cure: } 2.69 \% \text { XDR-TB, } \\
\text { cured: } 0.39\end{array}$ & Beta & {$[21,22]$} \\
\hline Lost to follow-up & Death & 6.87 & Beta & [24] \\
\hline \multirow[t]{2}{*}{ Sputum culture converted } & $\begin{array}{l}\text { Active secondary MDR-TB } \\
\text { (relapse) }\end{array}$ & 0.98 & Beta & [14] \\
\hline & MDR-TB or active XDR-TB (relapse) & (21.4 to XDR-TB; 78.6 to MDR-TB) & & [14] \\
\hline \multirow[t]{2}{*}{ Treatment completion and cured } & $\begin{array}{l}\text { Active secondary MDR-TB } \\
\text { (reoccurrence) }\end{array}$ & 0.20 & Beta & [21] \\
\hline & $\begin{array}{l}\text { MDR-TB or active XDR-TB } \\
\text { (reoccurrence) }\end{array}$ & (21.4 to XDR-TB; 78.6 to MDR-TB) & & [14] \\
\hline
\end{tabular}


plus BR, versus BR alone. This calculation generated a treatment-related hazard rate, which was subsequently used to estimate the probability of culture conversion while receiving add-on therapies (Table 2). The bedaquiline trial publication reported the hazard ratio for SCC versus placebo as 2.44 (95\% confidence interval $[\mathrm{CI}]$ : 1.57-3.80) [14].

For delamanid, comparative efficacy versus placebo was derived using a Bayesian meta-analysis. For the metaanalysis, a fixed effects model was fitted to aggregated summary data from the study by Gler and colleagues [12]. The Bayesian model consisted of a binomial likelihood function, complementary log-log link function, and vague prior distributions. The estimated hazard ratio for delamanid versus placebo was 1.73 (95\% CI: 1.153-2.627), based on a calculation of 1 divided by 0.58 (the reported hazard ratio for increased time to SCC with placebo vs. delamanid) [12]. Data for delamanid from Gler 2012 were only available for 8 weeks but in the model, the duration of add-on therapy for delamanid was assumed to be 24 weeks. In the absence of additional data, it was conservatively assumed that the hazard ratio for SCC at 8 weeks was maintained for 24 weeks.

Linezolid is used in an off-label setting for MDR-TB; data from a German observational study were used to determine relative efficacy for linezolid [13] (Table 2). In this study, patients were treated according to WHO recommendations, using fluoroquinolones, injectable agents and other second-line oral agents (BR), linezolid being one agent in this regimen. Patients who received linezolid in their BR were more likely to achieve SCC compared with those without linezolid (relative risk of 1.28; 95\% confidence interval 0.99-1.6), although the time taken to achieve SCC was significantly longer in the linezolid group (mean time to SCC of 102.9 days for linezolid versus 65.4 days without linezolid). The effect of linezolid was modelled using the relative risk of SCC, which was assumed to represent the effect of linezolid on time to SCC.

Treatment discontinuation with a novel or Group 5 intervention was simulated in terms of both loss to follow-up and discontinuation of the intervention due to AEs.
The treatment algorithm for patients with MDR-TB including drug treatment, dosages, and length-of-stay in isolated care - reflected recommendations made in local clinical guidelines and expert opinion [6-8, 25]. Surgery was excluded from this analysis as a treatment intervention, as guidelines recommend surgery in only a small minority of MDR-TB cases [6].

The health utility weights applied in the simulation model were adapted from a previous study in a low incidence setting [17], as local data for Germany are not available. Patients who occupied the active MDR-TB and lost to follow-up states were assigned the utility weight for active TB, while patients who occupied the sputum converted MDR-TB states were assigned a utility weight that was dependent on the time since conversion (a longer duration of sustained conversion was associated with an improvement in utility weight up to the utility weight for the general population [treatment completion]). Accordingly, the utility weight for sputum converted MDR-TB was estimated by linear interpolation of the weights for active TB (lower bound) and the general population (upper bound). All health utility weight inputs are summarized in Additional file 1: Table S1.

\section{Cost inputs}

Direct medical costs were assumed to consist of drug acquisition costs, costs of treatment monitoring, costs of administered care (inpatient and outpatient care), costs of end of life care, and costs of managing AEs.

Unit costs for each drug in the BR, the cost of each monitoring resource, outpatient visits, and inpatient costs were sourced from publicly available tariffs and formularies in Germany [26]. Drug costs $(€ / 2015)$ were based on the German Drug Directory (Lauer-Taxe Online 2015), and calculated based on the smallest pack available for the minimum period of treatment necessary, assuming use of generics when available [27]. The cost of resource use and monitoring was adapted from a previous study [11].

The cost of AEs included medication costs and monitoring costs, and was based on expert opinion and clinical study data [14] [analysis with data on file] [12, 28]. AEs that were at least potentially causally related to the

Table 2 Summary of relative efficacy of adding novel or group 5 interventions to a BR

\begin{tabular}{|c|c|c|c|c|}
\hline Intervention & Time to SCC & Mean treatment effect as Relative Risk RR (SE) & Discontinuation rate per month (\%) & Source/assumption \\
\hline $\begin{array}{l}\text { Bedaquiline vs. } \\
\text { placebo }\end{array}$ & 83 days vs. 125 days & $2.44(0.57)$ & $0.87 \%$ & [14] \\
\hline $\begin{array}{l}\text { Delamanid vs. } \\
\text { placebo }\end{array}$ & $\begin{array}{l}\text { Not reported (HR of } 0.58 \\
\text { reported only for placebo } \\
\text { vs. delamanid) }\end{array}$ & $1.73(0.38)$ & $1.26 \%$ & $\begin{array}{l}\text { Analysis of data } \\
\text { reported in [12] }\end{array}$ \\
\hline $\begin{array}{l}\text { Linezolid vs. } \\
\text { placebo }\end{array}$ & 103 days vs. 65 days & $1.28(0.57)$ & $3.1 \%$ & [13] see main text \\
\hline
\end{tabular}

$B R$ Background regimen, $R R$ relative risk, SE Standard Error, SCC sputum culture conversion 
investigational study drug and occurring in $>5 \%$ of patients were included for each intervention. Inputs for the cost and duration of treatment-associated AEs are presented in Additional file 2: Table S2. The cost of electrocardiogram and liver enzyme monitoring was absorbed within overall monitoring costs. A summary of costs for the total costs of treatment, split by interventions and cost category (hospitalisations, outpatient costs, medication costs etc.) is displayed in Additional file 3: Table S3.

\section{Sensitivity analysis}

Both probabilistic and deterministic sensitivity analyses were conducted following international recommendations [29]. In the deterministic simulations, key model parameters and assumptions were varied by $\pm 20 \%$, including clinical efficacy for each intervention, transition probabilities, utility weights, discount rates, the cost of AEs, and drug costs.

In probabilistic analyses, the likelihood of bedaquiline, linezolid, or delamanid plus BR being cost-effective versus BR alone was explored at different willingness-topay thresholds.

\section{Results}

\section{Base-case results}

Over the 10-year time horizon, the total discounted perpatient costs associated with the interventions under evaluation were $€ 85,575$ for bedaquiline, $€ 81,079$ for delamanid, $€ 80,460$ for linezolid plus BR, and $€ 60,962$ for BR only. Total costs were largely driven by TB drug costs and hospitalisation costs; costs associated with $\mathrm{AE}$ management were relatively small (Table 3 ).

The differing cost of BR treatment across regimens displayed in Table 3 primarily reflects two model assumptions. Firstly, patients who fail to achieve sputum culture conversion in the first 20 months of treatment are considered treatment failures, and go on to receive further BR treatment in the secondary MDR-TB state until cure, loss to follow-up, or death. With add-on therapies, more patients achieve cure, fewer patients fail treatment, and fewer patients go on to receive BR in the secondary MDR-TB state. Secondly, the model assumes a difference in the mortality rate between culture-converted and unconverted patients. The higher rate of cure with add-on therapies means that patients will on average live longer, and consequently, a higher percentage of patients will complete the full course of BR treatment.

The total discounted cost and discounted QALYs for the single cohort of 100 patients assigned to bedaquiline plus BR was $€ 8,557,529$ and 479 QALYs, respectively (Table 4). The total discounted cost and discounted QALYs for the patients assigned to delamanid plus BR was $€ 8,107,888$ and 421 QALYs, respectively, and to linezolid plus BR, €8,045,981 and 391 QALYs, respectively. In terms of life-years gained (LYG), the total discounted LYG for the patients assigned to bedaquiline plus BR was 595; delamanid plus BR, 536; to linezolid plus BR, 507; and BR alone, 482 (Table 4).

The incremental cost per QALY gained versus BR alone was $€ 22,238$ for bedaquiline, $€ 38,703$ for delamanid, and $€ 87,484$ for linezolid (Table 4).

The results of the fully incremental analysis are presented graphically using the cost-efficiency frontier (Fig. 2), which compares the expected costs ( $x$-axis) and benefits (y-axis) of each intervention in the evaluation [30]. The cost-efficiency frontier is plotted by connecting those treatments that represent the most cost-efficient use of healthcare resources, relative to any other therapy or their combinations.

In this analysis, BR alone and bedaquiline plus BR were considered the most cost-efficient interventions, with delamanid plus BR and linezolid plus BR being dominated by a combination of BR alone with or without bedaquiline. By excluding delamanid and linezolid

Table 3 Per-patient and population level costs for the interventions included in the analysis

\begin{tabular}{|c|c|c|c|c|c|c|c|}
\hline \multicolumn{8}{|l|}{ Patient-level } \\
\hline $\begin{array}{l}\text { Treatment } \\
\text { strategy }\end{array}$ & $\begin{array}{l}\text { Added costs for a novel } \\
\text { or group } 5 \text { intervention }(€)\end{array}$ & $\begin{array}{l}\text { Cost of BR } \\
\text { treatment }\end{array}$ & $\begin{array}{l}\text { Hospitalization } \\
\text { costs }(€)\end{array}$ & $\begin{array}{l}\text { Outpatient } \\
\text { care }(€)\end{array}$ & $\begin{array}{l}\text { Monitoring } \\
\text { costs }(€)\end{array}$ & $\begin{array}{l}\text { Adverse event costs of } \\
\text { group } 5 \text { drugs }(€)\end{array}$ & Total $(€)$ \\
\hline Bedaquiline plus BR & 30,799 & 28,652 & 24,038 & 98 & 1970 & 17 & 85,575 \\
\hline Delamanid plus BR & 22,829 & 29,626 & 26,362 & 108 & 2152 & 2 & 81,079 \\
\hline Linezolid plus BR & 20,302 & 29,968 & 27,392 & 107 & 2569 & 121 & 80,460 \\
\hline BR only & 0 & 30,270 & 28,180 & 119 & 2393 & - & 60,962 \\
\hline \multicolumn{8}{|c|}{ Population-level, 100 patients with MDR-TB } \\
\hline Bedaquiline plus BR & $3,079,915$ & $2,865,240$ & $2,403,791$ & 9833 & 197,014 & 1735 & $8,557,529$ \\
\hline Delamanid plus BR & $2,282,920$ & $2,962,563$ & $2,636,180$ & 10,755 & 215,241 & 229 & $8,107,888$ \\
\hline Linezolid plus BR & $2,030,217$ & $2,996,842$ & $2,739,250$ & 10,715 & 256,867 & 12,091 & $8,045,981$ \\
\hline BR only & 0 & $3,026,959$ & $2,817,995$ & 11,919 & 239,279 & - & $6,096,152$ \\
\hline
\end{tabular}

$A E$ adverse event, $B R$ background regimen, $T B$ tuberculosis, MDR-TB multidrug-resistant tuberculosis 
Table 4 Incremental cost per QALY gained

\begin{tabular}{|c|c|c|c|c|c|c|c|c|c|}
\hline \multirow{2}{*}{$\begin{array}{l}\text { Treatments } \\
\text { ordered from least } \\
\text { to most effective }\end{array}$} & \multicolumn{3}{|c|}{ Patient-level } & \multicolumn{3}{|c|}{ Population-level (100 MDR patients) } & \multicolumn{3}{|c|}{ Incremental cost per QALY gained $(€)$} \\
\hline & $\begin{array}{l}\text { Total } \\
\text { cost, } €\end{array}$ & $\begin{array}{l}\text { Total QALYS } \\
\text { gained }\end{array}$ & $\begin{array}{l}\text { Total } \\
\text { LYG }\end{array}$ & $\begin{array}{l}\text { Total } \\
\text { cost, } €\end{array}$ & $\begin{array}{l}\text { Total QALYS } \\
\text { gained }\end{array}$ & $\begin{array}{l}\text { Total } \\
\text { LYG }\end{array}$ & $\begin{array}{l}\text { Versus BR } \\
\text { alone }\end{array}$ & $\begin{array}{l}\text { Versus Linezolid } \\
\text { plus BR }\end{array}$ & $\begin{array}{l}\text { Versus delamanid } \\
\text { plus BR }\end{array}$ \\
\hline BR alone & 60,962 & 3.68 & 4.82 & $6,096,152$ & 368 & 482 & - & 87,484 & 38,703 \\
\hline Linezolid plus BR & 80,460 & 3.91 & 5.07 & $8,045,981$ & 391 & 507 & 87,484 & - & 2026 \\
\hline Delamanid plus BR & 81,079 & 4.21 & 5.36 & $8,107,888$ & 421 & 536 & 38,703 & 2026 & - \\
\hline Bedaquiline plus BR & 85,575 & 4.79 & 5.95 & $8,557,529$ & 479 & 595 & 22,238 & 5787 & 7774 \\
\hline
\end{tabular}

$B R$ Background Regimen, QALY Quality-Adjusted Life Year, LYG life-years gained

from further consideration in the cost-efficiency frontier, the analysis shows that treatment with BR alone is the most cost-efficient therapy at willingness to pay thresholds up to $€ 22,000$ per QALY gained, with bedaquiline plus BR becoming the most cost-efficient therapy at thresholds greater than $€ 22,000$ (which falls below the informal threshold of $€ 30,000$ to $€ 50,000$ per QALY commonly applied in European healthcare systems).

\section{Sensitivity analyses}

The results of the deterministic sensitivity analysis are presented in Table 5.

In the one-way sensitivity analysis, the effect of bedaquiline on SCC was the most influential parameter, with ICERs of $€ 17,711$ (plus $20 \%$ more effective) and $€ 30,960$ (20\% less effective) in comparisons with BR alone. Other influential parameters included the rate of SCC for BR alone (ICERs of between $€ 20,150$ [20\% higher rate of SCC] and $€ 26,384$ [ $20 \%$ lower rate of SCC]), and the utility assigned to cured patients (ICER of $€ 18,770$ for perfect health post-cure versus $€ 22,238$ in the base case). The model results were not sensitive to assumptions on discounting rates, the cost of BR medication, the cost of AEs, or the rate of relapse after cure.

In comparisons of bedaquiline plus BR versus linezolid plus BR, all ICERs were below $€ 15,000$ per QALY gained. In the analysis where the duration of linezolid was reduced from 42 to 24 weeks (same duration as with bedaquiline plus $\mathrm{BR}$ ), the ICER comparing bedaquiline plus BR versus linezolid plus BR increased from $€ 5778$ (base case) to $€ 13,553$ per QALY gained, which remains below a threshold of $€ 30,000$ per QALY, which is commonly applied informally in European healthcare systems. In comparisons of bedaquiline plus BR versus delamanid plus BR, all ICERs were below $€ 20,000$ per QALY gained.

The results of the probabilistic sensitivity analysis are presented in Fig. 3. All data on probabilistic distributions, parameters and definitions used in the probabilistic sensitivity analysis are shown in Additional file 4: Table S4.

The probability that bedaquiline plus BR was the most cost-effective treatment strategy at a willingness to pay threshold of $€ 30,000$ was $54.5 \%$, versus $22.9 \%$ for BR alone, $4.4 \%$ for linezolid plus BR and $18.2 \%$ for delamanid plus BR. At a higher threshold of $€ 50,000$ per QALY gained (informally, towards the upper limit of willingnessto-pay thresholds applied in European healthcare systems for orphan drugs), the probability that bedaquiline plus $\mathrm{BR}$ is a cost-effective alternative to existing interventions was greater than $70 \%$.

\section{Discussion}

The results of this study demonstrate that, in the German setting, adding bedaquiline, delamanid, or linezolid to a

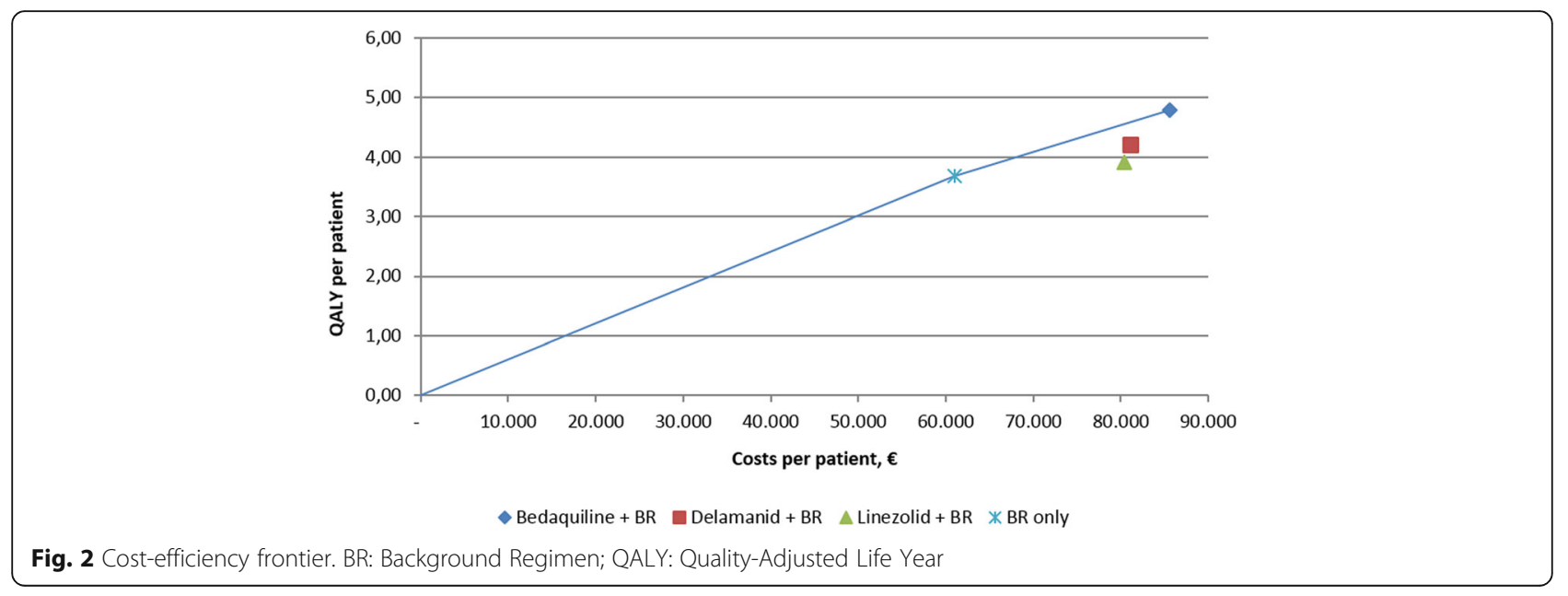


Table $\mathbf{5}$ One-way sensitivity analysis (per patient level)

\begin{tabular}{|c|c|c|c|c|c|c|c|c|}
\hline \multirow[t]{2}{*}{ Parameter } & \multirow[t]{2}{*}{ Variation } & \multicolumn{4}{|c|}{ Cost / QALY } & \multicolumn{3}{|c|}{$\begin{array}{l}\text { Incremental cost per QALY gained } \\
(€) \text {, Bedaquiline }\end{array}$} \\
\hline & & $\begin{array}{l}\text { BR } \\
\text { alone }\end{array}$ & $\begin{array}{l}\text { Linezolid } \\
\text { plus BR }\end{array}$ & $\begin{array}{l}\text { Delamanid } \\
\text { plus BR }\end{array}$ & $\begin{array}{l}\text { Bedaquiline } \\
\text { plus BR }\end{array}$ & $\begin{array}{l}\text { Versus } \\
\text { BR alone }\end{array}$ & $\begin{array}{l}\text { Versus } \\
\text { Linezolid plus } \\
\text { BR }\end{array}$ & $\begin{array}{l}\text { Versus } \\
\text { Delamanid } \\
\text { plus BR }\end{array}$ \\
\hline Base case & & $\begin{array}{l}60,962 / \\
3.68\end{array}$ & $\begin{array}{l}80,460 / \\
3.91\end{array}$ & $\begin{array}{l}81,079 / \\
4.21\end{array}$ & $\begin{array}{l}85,575 / \\
4.79\end{array}$ & 22,238 & 5787 & 7774 \\
\hline \multirow[t]{2}{*}{$\begin{array}{l}\text { BR SCC rates at 6- } \\
\text { months }\end{array}$} & $+20 \%(37.2 \%$ SCC to $44.6 \%)$ & $\begin{array}{l}58,648 / \\
4.00\end{array}$ & $\begin{array}{l}78,179 / \\
4.24\end{array}$ & $\begin{array}{l}78,192 / \\
4.58\end{array}$ & $\begin{array}{l}82,170 / \\
5.17\end{array}$ & 20,105 & 4316 & 6713 \\
\hline & $-20 \%(37.2 \%$ SCC to $29.7 \%)$ & $\begin{array}{l}63,037 / \\
3.36\end{array}$ & $\begin{array}{l}82,518 / \\
3.56\end{array}$ & $\begin{array}{l}83,807 / \\
3.82\end{array}$ & $\begin{array}{l}89,010 / \\
4.35\end{array}$ & 26,384 & 8196 & 9802 \\
\hline \multirow[t]{2}{*}{$\begin{array}{l}\text { Effect of bedaquiline on } \\
\text { SCC rates }\end{array}$} & $+20 \%$ & $\begin{array}{l}60,962 / \\
3.68\end{array}$ & $\begin{array}{l}80,460 / \\
3.91\end{array}$ & $\begin{array}{l}81,079 / \\
4.21\end{array}$ & $\begin{array}{l}84,384 / \\
5.01\end{array}$ & 17,711 & 3569 & 4162 \\
\hline & $-20 \%$ & $\begin{array}{l}60,962 / \\
3.68\end{array}$ & $\begin{array}{l}80,460 / \\
3.91\end{array}$ & $\begin{array}{l}81,079 / \\
4.21\end{array}$ & $\begin{array}{l}86,986 / \\
4.52\end{array}$ & 30,960 & 10,565 & 18,921 \\
\hline $\begin{array}{l}\text { Maximum duration of } \\
\text { linezolid treatment }\end{array}$ & $\begin{array}{l}42 \text { to } 24 \text { weeks (duration of } \\
\text { Bedaquiline treatment) }\end{array}$ & $\begin{array}{l}60,962 / \\
3.68\end{array}$ & $\begin{array}{l}73,307 / \\
3.88\end{array}$ & $\begin{array}{l}81,079 / \\
4.21\end{array}$ & $\begin{array}{l}85,575 / \\
4.79\end{array}$ & 22,238 & 13,553 & 7774 \\
\hline \multirow[t]{2}{*}{ Relapse after cure } & $+20 \%$ & $\begin{array}{l}61,232 / \\
3.65\end{array}$ & $\begin{array}{l}80,732 / \\
3.87\end{array}$ & $\begin{array}{l}81,421 / \\
4.16\end{array}$ & $\begin{array}{l}85,716 / \\
4.77\end{array}$ & 21,809 & 5508 & 7092 \\
\hline & $-20 \%$ & $\begin{array}{l}60,684 / \\
3.72\end{array}$ & $\begin{array}{l}80,180 / \\
3.95\end{array}$ & $\begin{array}{l}80,729 / \\
4.26\end{array}$ & $\begin{array}{l}85,433 / \\
4.81\end{array}$ & 22,701 & 6094 & 8552 \\
\hline \multirow[t]{2}{*}{$\begin{array}{l}\text { Utility weight for no } \\
\text { cure }\end{array}$} & plus $20 \%$ & $\begin{array}{l}60,962 / \\
3.95\end{array}$ & $\begin{array}{l}80,460 / \\
4.16\end{array}$ & $\begin{array}{l}81,079 / \\
4.46\end{array}$ & $\begin{array}{l}85,575 / \\
5.02\end{array}$ & 22,988 & 5983 & 8056 \\
\hline & $-20 \%$ & $\begin{array}{l}60,962 / \\
3.48\end{array}$ & $\begin{array}{l}80,460 / \\
3.72\end{array}$ & $\begin{array}{l}81,079 / \\
4.04\end{array}$ & $\begin{array}{l}85,575 / \\
4.66\end{array}$ & 20,973 & 5465 & 7337 \\
\hline Utility for cure & Perfect health after cure & $\begin{array}{l}60,962 / \\
4.26\end{array}$ & $\begin{array}{l}80,460 / \\
4.53\end{array}$ & $\begin{array}{l}81,079 / \\
4.89\end{array}$ & $\begin{array}{l}85,575 / \\
5.57\end{array}$ & 18,770 & 4905 & 6596 \\
\hline \multirow[t]{4}{*}{ Discount rate } & $0 \%$ cost $3 \%$ outcomes & $\begin{array}{l}62,032 / \\
3.68\end{array}$ & $\begin{array}{l}81,524 / \\
3.91\end{array}$ & $\begin{array}{l}82,098 / \\
4.21\end{array}$ & $\begin{array}{l}86,538 / \\
4.79\end{array}$ & 22,141 & 5672 & 7676 \\
\hline & $6 \%$ costs $3 \%$ outcomes & $\begin{array}{l}60,039 / \\
3.68\end{array}$ & $\begin{array}{l}79,547 / \\
3.91\end{array}$ & $\begin{array}{l}80,210 / \\
4.21\end{array}$ & $\begin{array}{l}84,765 / \\
4.79\end{array}$ & 22,340 & 5903 & 7875 \\
\hline & $3 \%$ costs $0 \%$ outcomes & $\begin{array}{l}60,962 / \\
4.12\end{array}$ & $\begin{array}{l}80,460 / \\
4.38\end{array}$ & $\begin{array}{l}81,079 / \\
4.73\end{array}$ & $85,575 / 5.4$ & 19,224 & 5015 & 6712 \\
\hline & $3 \%$ costs $6 \%$ outcomes & $\begin{array}{l}60,962 / \\
3.33\end{array}$ & $\begin{array}{l}80,460 / \\
3.52\end{array}$ & $\begin{array}{l}81,079 / \\
3.79\end{array}$ & $\begin{array}{l}85,575 / \\
4.29\end{array}$ & 25,473 & 6611 & 8914 \\
\hline \multirow[t]{2}{*}{ Cost for BR medication } & $+20 \%$ & $\begin{array}{l}66,824 / \\
3.68\end{array}$ & $\begin{array}{l}86,271 / \\
3.91\end{array}$ & $\begin{array}{l}86,831 / \\
4.21\end{array}$ & $\begin{array}{l}91,177 / \\
4.79\end{array}$ & 22,003 & 5550 & 7514 \\
\hline & $-20 \%$ & $\begin{array}{l}55,099 / \\
3.68\end{array}$ & $\begin{array}{l}74,649 / \\
3.91\end{array}$ & $\begin{array}{l}75,327 / \\
4.21\end{array}$ & $\begin{array}{l}79,974 / \\
4.79\end{array}$ & 22,474 & 6024 & 8034 \\
\hline \multirow[t]{2}{*}{ Cost for AE's } & $+20 \%$ & $\begin{array}{l}60,962 / \\
3.68\end{array}$ & $\begin{array}{l}80,484 / \\
3.91\end{array}$ & $\begin{array}{l}81,079 / \\
4.21\end{array}$ & $\begin{array}{l}85,579 / \\
4.79\end{array}$ & 22,242 & 5764 & 7779 \\
\hline & $-20 \%$ & $\begin{array}{l}60,962 / \\
3.68\end{array}$ & $\begin{array}{l}80,436 / \\
3.91\end{array}$ & $\begin{array}{l}81,078 / \\
4.21\end{array}$ & $\begin{array}{l}85,572 \text { / } \\
4.79\end{array}$ & 22,235 & 5811 & 7768 \\
\hline
\end{tabular}

AE Adverse event, BR Background regimen, QALY Quality-adjusted life-year, SCC Sputum culture conversion

BR would lead to QALY and LY gains under a range of scenarios. The cost per QALY versus BR alone ranged from $€ 22,238$ for bedaquiline, to $€ 38,703$ for delamanid, and $€ 87,484$ for linezolid.

QALY gains for add-on therapies to a BR were driven by improved efficacy versus BR alone. As would be expected from adding interventions to a BR, higher total costs associated with add-on therapies were driven by increased drug acquisition costs, although total incremental costs were partially offset by reduced hospitalisation costs (resulting from improved sputum culture conversion compared with BR alone and therefore assuming less time spent in the inpatient setting). Monitoring costs and the costs of AE management had a relatively limited effect on the outcome, as the AEs were generally treatable with inexpensive medications.

Where multiple treatment options are available, it is important to identify which strategies provide the most efficient use of limited health care resources. This is achieved through fully incremental analysis where the 


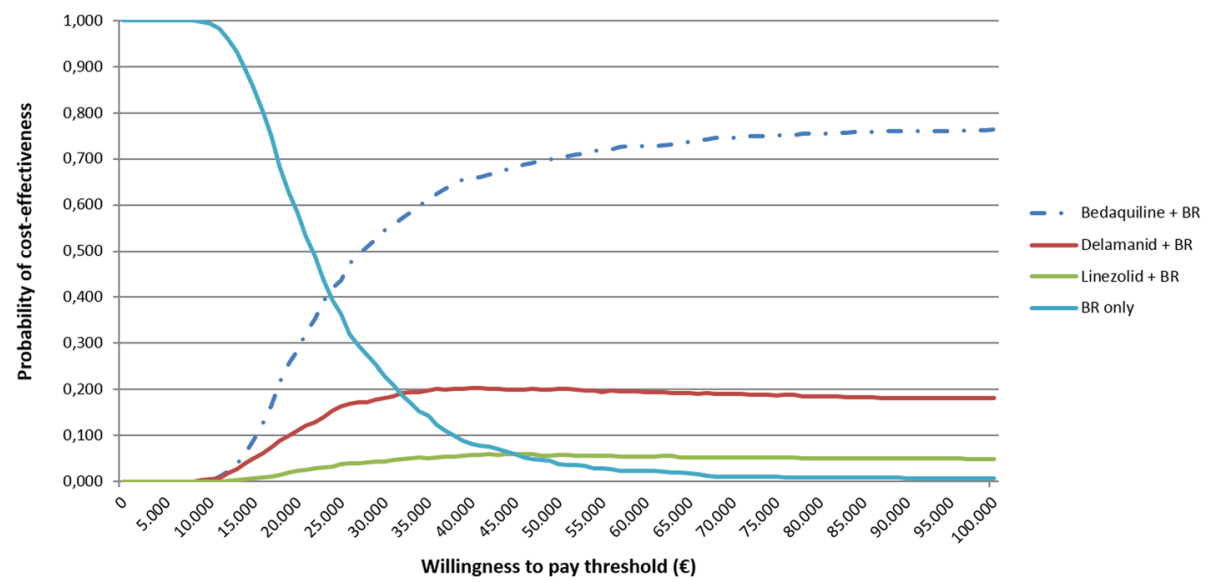

Fig. 3 Multiway cost-effectiveness acceptability curve. BR: Background regimen

cost-effectiveness of transitioning from one option to a more effective option is assessed. A key conclusion from this analysis was that a mix of strategies comprising BR alone and BR plus bedaquiline may yield greater QALY benefits than treatment with either linezolid or delamanid, but at higher total costs. More formally, linezolid and delamanid were dominated by a combination of BR alone and BR plus bedaquiline, and consequently, the administration of linezolid and delamanid would be considered a cost-inefficient use of available resources. Following standard practice [31], linezolid and delamanid were excluded from further cost-effectiveness analyses. The resulting analyses suggest that BR plus bedaquiline is the most cost-efficient treatment strategy at thresholds greater than $€ 22,000$ per QALY gained.

At the same time, the exclusion of linezolid and delamanid due to cost would not be clinically justifiable, given that dominance can only be achieved if some patients receive BR alone. Without access to novel therapies, some of these patients may go on to develop further drug resistance because of inadequate treatment with BR, possibly leading to active secondary MDR-TB with an excess morbidity and mortality burden. Moreover, there is a recognition that combining several new mechanisms of action will likely further enhance treatment outcomes [32]. Thus, MDR-TB treatment strategies should not be excluded based on dominance, given the severe consequences of poor outcomes in this population with a BR-only based treatment.

Previous studies have focused on the comparative effectiveness [33] or the cost-effectiveness of novel or Group 5 interventions compared with BR alone [15-18], but this is the first study to explicitly model costeffectiveness across novel or Group 5 interventions for the treatment of MDR-TB within a consistent model structure. The results of this analysis are consistent with previous cost-effectiveness analyses of bedaquiline for
MDR-TB [15, 17, 34], reinforcing that this intervention remains cost-effective under a range of assumptions and different country settings. The total costs for BR alone and ICER versus bedaquiline varied slightly to values reported in a previous cost-effectiveness analysis [34], due to a minor model variation in which treatment efficacy was linked to discontinuation over time: patients who leave the model (after failure to successfully treat active secondary MDR-TB) are assumed to derive no further benefit from treatment. Nevertheless, the conclusions are broadly consistent.

The results of the delamanid analysis presented here differ from a previous analysis by Diel and colleagues [18]. In the current analysis, delamanid plus BR was associated with an ICER of $€ 38,703$ per QALY versus BR alone, whereas in the Diel et al. publication, delamanid dominated over BR with an ICER of $€-3494$ per QALY gained. This discrepancy is very likely to reflect the different perspectives considered in these analyses: societal in the Diel analysis; payer in the current analysis, as well as the use of different data sources to inform the modelling by using patient-level data and the differing definitions of outcome parameters. To our knowledge, no economic evaluations of linezolid in MDR-TB have been published to date.

Due to the toxicity associated with MDR-TB therapies, it is important to consider the impact of AEs and their time of onset when comparing treatment options. This is the first study to comprehensively incorporate the costs of AEs associated with Group 5 interventions into a cost-effectiveness analysis of treatments for MDR-TB. It was not possible to assign utility weights to these AEs due to the lack of disease-specific data to inform utility loss associated with MDR-TB in the German setting. Although proxy data could - in theory - be substituted to inform utility loss, attempting to apply such data on top of a utility weight already heavily impacted by disutility due to BR side effects proved unfeasible in this analysis. 
The development of utility weights for MDR-TB specific AEs would therefore be valuable to inform future cost-effectiveness analyses in MDR-TB. However, due to the comparative tolerability of the interventions considered in this analysis relative to other MDR-TB drugs, the conclusions are likely to hold even if such disutility were to be explicitly modelled. Alternatively, a qualityadjusted time without symptoms or toxicity (Q-TWIST) approach [35] could be considered. Although typically applied in oncology, such an analysis would capture disease- and treatment-related disutility, and would also allow the user to model the patient impact of the muchneeded shortened treatment regimens that are currently in clinical trials.

Additional future modelling work may account for recent developments in the MDR-TB treatment landscape, looking at shorter simplified regimens or different combinations of new and current drugs. Trials are currently underway to assess the efficacy of innovative combinations of Group 5 or novel interventions, such as the 6-month combination regimen of bedaquiline, linezolid, and PA-824 being evaluated in the NiX-TB-(B-L-Pa) trial (NCT02333799) [36], as well as the STREAM study. The STREAM study is a Phase 3, multicentre, international randomised controlled trial aiming to assess the safety and efficacy of shorter MDR-TB regimens [37]. The second stage of the STREAM study will include two bedaquiline-containing arms [38], including an all-oral regimen. Further, the EndTB program aims to expand access to new TB drugs such as bedaquiline and delamanid in 16 countries [39], while an NIH-sponsored safety trial is planned in South Africa.

The analysis presented here was subject to a number of assumptions, which have been described, discussed, and justified in detail previously [17]. Briefly, the use of trial data for bedaquiline and delamanid may not accurately reflect results found in German clinical practice. However, the results were not found to differ substantially when interim data from real-world evidence studies from a European and African setting were applied in sensitivity analyses [40-42], and therefore, the results of this analysis can be assumed to be applicable in the realworld setting.

Secondly, the mortality imbalance observed in the bedaquiline $\mathrm{C} 208$ clinical trial was not captured here because no causal link to study medication was found. Further, newly published results of the C209 trial [43] as well as recent published interim data from the ongoing Compassionate Use program for bedaquiline in France, South Africa, and Latvia [40-42, 44], suggest a lower mortality rate than that observed in the C208 trial, justifying this assumption.

The third assumption relates to patients after leaving the model. Patients who were lost to follow-up were assumed to remain in this state until death and did not incur any costs, for the purpose of simplifying the model structure. Data are limited on the retreatment of such patients [45], and due consideration to treatment with third-line or even fourth-line regimens was beyond the scope of this analysis. Regardless, this assumption is unlikely to substantially impact the results of the analysis, given that the rate of loss to follow-up was assumed to be consistent between treatment strategies [17].

In addition, patients who achieved sputum culture conversion did not experience any lasting disutility and were assigned the same utility as patients in the general population, which is likely to be a simplistic assumption. However, data on the utility of patients who have been cured of MDR-TB are limited and in sensitivity analyses, the impact of varying utility weights for the 'cured' health state had a relatively small effect on the ICER.

Finally, heterogeneity between the studies considered in this analysis introduces an element of uncertainty, and can be considered a key limitation of the model structure. Relative efficacy outputs should be interpreted within the appropriate context, especially given that the hazard ratio of SCC was the most influential parameter in one-way sensitivity analyses.

Although the best available evidence was used at the time of model development (clinical trial data for bedaquiline and delamanid; German observational data for linezolid due to a lack of RCTs in MDR-TB), other studies exist that could be used to model relative efficacy. For example, clinical trials carried out in XDR-TB populations in the Chinese and Korean settings [46-49], respectively, demonstrated higher rates of sputum culture conversion for linezolid than were reported in the German study used in this analysis [13]. In addition, recent contributions to the literature reporting MDR-TB and XDR-TB outcomes for bedaquiline [43] and XDRTB outcomes for delamanid [50] could also be considered in future research. Alternatively, the collection and application of direct comparative clinical trial data would allow for treatment effect to be modelled more objectively.

\section{Conclusions}

The addition of bedaquiline, delamanid, or linezolid to a BR would result in QALY gains over BR alone when applied in the German healthcare system. Bedaquiline is likely to be the most cost-effective intervention for the treatment of MDR-TB, when added to a BR regimen at thresholds greater than $€ 22,000$ per QALY. These results may be used to inform MDR-TB treatment and reimbursement decisions within the German healthcare system and in other high-income countries. 


\section{Additional files}

Additional file 1: Table S1. Adverse events considered in the economic model $[12,14,28,51,52]$. (DOCX 22 kb)

Additional file 2: Table S2. Utility data used for health states in the economic model. (DOCX $13 \mathrm{~kb}$ )

Additional file 3: Table S3. Total costs of treatment in the MDR-TB cohort, split by intervention \& cost category. (DOCX $13 \mathrm{~kb}$ )

Additional file 4: Table S4. Probabilistic distributions, parameters and definitions as used in the PSA. (DOCX $14 \mathrm{~kb}$ )

\section{Abbreviations}

AE: Adverse event; BR: Background regimen; Cl: Confidence interval; ICER: Incremental cost-effectiveness ratio; LYG: Life-year gained; MDRTB: Multidrug-resistant tuberculosis; QALY: Quality-adjusted life-year; QTWIST: Quality-adjusted time without symptoms or toxicity; SCC: Sputum culture conversion; TB: Tuberculosis; XDR: Extensively drug-resistant

\section{Acknowledgements}

The authors would like to thank Elizabeth Griffiths and Jasim Uddin at PAREXEL International for their help in the preparation of this manuscript.

\section{Funding}

This study was commissioned and funded by Janssen Pharmaceutical Companies of Johnson and Johnson. D. Wirth and R. Dass were employed by Janssen-Cilag at the time of writing this manuscript. R. Hettle is employed by PAREXEL International. Janssen-Cilag provided financial support to PAREXEL International for writing and modelling contributions. The funders played a role $\mathrm{n}$ the study design, data collection and analysis, decision to publish, and preparation of the manuscript. Janssen-Cilag and PAREXEL International provided support in the form of salaries for the authors. The specific roles of these authors are articulated in the author contributions section.

\section{Availability of data and materials}

The datasets used to develop the model presented in the current study are available from the corresponding author on reasonable request.

\section{Authors' contributions}

DW contributed to the conception and design of this study, data acquisition for model inputs, and data analysis. RD contributed to the conception and design of this study, and data analysis. $\mathrm{RH}$ designed the model that this study is based on, and generated the model results.

All authors were involved in drafting the manuscript content, and all authors read and approved the final manuscript. The authors jointly made the decision to publish and agree to be accountable for all aspects of this study. They all comply with the International Committee of Medical Journal Editors guidelines for authorship.

\section{Competing interests}

This study was financially supported by Janssen-Cilag, and publication was not contingent on the approval or censorship of the manuscript by JanssenCilag. D. Wirth and R. Dass were employed by Janssen-Cilag at the time of writing this manuscript; R. Hettle received fees from Janssen-Cilag in relation to the development of this manuscript and the model that it is based on. There are no patents or further conflicts of interest to declare.

\section{Consent for publication}

Not applicable.

\section{Ethics approval and consent to participate}

Not applicable.

\section{Author details}

${ }^{1}$ Health Economics \& Market Access, Janssen-Cilag GmbH, Johnson \& Johnson Platz 1, 41470 Neuss, Germany. ${ }^{2}$ Janssen-Cilag Ltd, High Wycombe, UK. ${ }^{3}$ PAREXEL International, London, UK.
Received: 9 September 2016 Accepted: 24 February 2017

Published online: 08 March 2017

\section{References}

1. WHO. Global Tuberculosis Report, 2014. http://apps.who.int/iris/bitstream/ 10665/137094/1/9789241564809_eng.pdf?ua=1. Accessed 5 Dec 2016.

2. ECDC. Tuberculosis surveillance and monitoring in Europe, 2015. http:// ecdc.europa.eu/en/publications/Publications/tuberculosis-surveillancemonitoring-Europe-2015.pdf. Accessed 5 Dec 2016.

3. Robert Koch Institut. Bericht zur Epidemiologie der Tuberkulose in Deutschland für 2013. http://www.rki.de/DE/Content/InfAZ/T/Tuberkulose/ Download/TB2013.pdf?_blob=publicationFile. Accessed 5 Dec 2016.

4. World Health Organisation. World Health Organisation, regional office for Europe: antimicrobial resistance. 2015. http://www.euro.who.int/en/healthtopics/disease-prevention/antimicrobial-resistance/antimicrobial-resistance Accessed 5 Dec 2016.

5. WHO. Guidelines for the programmatic management of drug-resistant tuberculosis. 2013. http://www.who.int/tb/challenges/mdr/programmatic_ guidelines_for_mdrtb/en/. Accessed 5 Dec 2016.

6. Lange C, Abubakar I, Alffenaar JW, Bothamley G, Caminero JA, Carvalho AC, et al. Management of patients with multidrug-resistant/extensively drug-resistant tuberculosis in Europe: a TBNET consensus statement. Eur Respir J. 2014:44:23-63.

7. Schaberg T, Bauer T, Castell S, Dalhoff K, Detjen A, Diel R, et al. Recommendation for therapy, chemoprevention and chemoprophylaxis of tuberculosis in adults and children. German Central Committee against Tuberculosis (DZK), German Respiratory Society (DGP). Pneumologie. 2012;66:133-71.

8. Schaberg T, Otto-Knapp R, Bauer T. New drugs for the treatment of Multidrug-resistant Tuberculosis (MDR-TB). Pneumologie. 2015;69:282-6.

9. Karo B, Hauer B, Hollo V, van der Werf MJ, Fiebig L, Haas W. Tuberculosis treatment outcome in the European Union and European Economic Area: an analysis of surveillance data from 2002-2011. Euro Surveill. 2015;20(49).

10. Robert Koch Institut. Bericht zur Epidemiologie der Tuberkulose in Deutschland für 2014. 2015. http://www.rki.de/DE/Content/InfAZ/T/ Tuberkulose/Download/TB2014.pdf?_blob=publicationFile. Accessed 5 Dec 2016

11. Diel R, Nienhaus A, Lampenius N, Rusch-Gerdes S, Richter E. Cost of multi drug resistance tuberculosis in Germany. Respir Med. 2014;108:1677-87.

12. Gler MT, Skripconoka V, Sanchez-Garavito E, Xiao H, Cabrera-Rivero JL, Vargas-Vasquez DE, et al. Delamanid for multidrug-resistant pulmonary tuberculosis. N Engl J Med. 2012:366:2151-60.

13. Migliori GB, Eker B, Richardson MD, Sotgiu G, Zellweger JP, Skrahina A, et al. A retrospective TBNET assessment of linezolid safety, tolerability and efficacy in multidrug-resistant tuberculosis. Eur Respir J. 2009:34:387-93.

14. Diacon AH, Pym A, Grobusch MP, de los Rios JM, Gotuzzo E, Vasilyeva I, et al. Multidrug-resistant tuberculosis and culture conversion with bedaquiline. $\mathrm{N}$ Engl J Med. 2014;371:723-32.

15. WHO, Vassell A. Cost-effectiveness of introducing bedaquiline in MDR-TB regimens - an exploratory analysis. 2013

16. Wolfson LJ, Gibbert J, Wirth D, Diel R. Cost-effectiveness of incorporating bedaquiline into a treatment regimen for multidrug-resistant/extensively drug-resistant tuberculosis in Germany. Eur Respir J. 2015;46(6):1826-9.

17. Wolfson LJ, Walker A, Hettle R, Lu X, Kambili C, Murungi A, et al. Costeffectiveness of adding bedaquiline to drug regimens for the treatment of multidrug-resistant tuberculosis in the UK. PLoS One. 2015:10:e0120763.

18. Diel R, Hittel N, Schaberg T. Cost effectiveness of treating multi-drug resistant tuberculosis by adding Deltyba to background regimens in Germany. Respir Med. 2015;109:632-41.

19. IQWiG. Arbeitspapier Kostenbestimmung. 2009. https://www.iqwig.de/ download/Arbeitspapier_Kostenbestimmung_v_1_0.pdf . Accessed 5 Dec 2016

20. WHO. Definitions and reporting framework for tuberculosis - 2013 revision. 2013. http://apps.who.int/iris/bitstream/10665/79199/1/9789241505345 eng.pdf . Accessed 5 Dec 2016.

21. Lee J, Lim HJ, Cho YJ, Park YS, Lee SM, Yang SC, et al. Recurrence after successful treatment among patients with multidrug-resistant tuberculosis. Int J Tuberc Lung Dis. 2011;15:1331-3.

22. Tiemersma EW, van der Werf MJ, Borgdorff MW, Williams BG, Nagelkerke NJ. Natural history of tuberculosis: duration and fatality of untreated pulmonary tuberculosis in HIV negative patients: a systematic review. PLoS One. 2011;6: e17601. 
23. Somocurcio JG, Sotomayor A, Shin S, Portilla S, Valcarcel M, Guerra D, et al. Surgery for patients with drug-resistant tuberculosis: report of 121 cases receiving community-based treatment in Lima, Peru. Thorax. 2007;62:416-21.

24. Franke MF, Appleton SC, Bayona J, Arteaga F, Palacios E, Llaro K, et al. Risk factors and mortality associated with default from multidrug-resistant tuberculosis treatment. Clin Infect Dis. 2008:46:1844-51.

25. WHO interim policy guidance. The use of bedaquiline in the treatment of multidrug resistant tuberculosis. 2013. http://apps.who.int/iris/bitstream/ 10665/84879/1/9789241505482_eng.pdf. Accessed 5 Dec 2016.

26. Institut für das Entgeltsystem im Krankenhaus. Definitionshandbuch G-DRGVersion 2013/2015 [Online]. http://www.g-drg.de/cms/G-DRG-System_2015/ Definitionshandbuch/Definitionshandbuch_2015. Accessed 5 Dec 2016.

27. Schwabe U, Paffrath D. Arzneiverordnungs-Report 2014: Aktuelle Daten, Kosten, Trends und Kommentare. Berlin: Springer; 2014. https://dx.doi.org/ 10.1007/978-3-662-43487-1. Accessed 5 Dec 2016.

28. Zhang X, Falagas ME, Vardakas KZ, Wang R, Qin R, Wang J, et al. Systematic review and meta-analysis of the efficacy and safety of therapy with linezolid containing regimens in the treatment of multidrug-resistant and extensively drug-resistant tuberculosis. J Thorac Dis. 2015;7:603-15.

29. Briggs AH, Weinstein MC, Fenwick EA, Karnon J, Sculpher MJ, Paltiel AD. Model parameter estimation and uncertainty analysis: a report of the ISPORSMDM Modeling Good Research Practices Task Force Working Group-6. Med Decis Making. 2012;32:722-32.

30. IQWiG. General Methods 4.2, 22 Apr 2015. https://www.iqwig.de/download/ IQWiG_General_Methods_Version_\%204-2.pdf . Accessed 5 Dec 2016.

31. Bala MV, Zarkin GA. Application of cost-effectiveness analysis to multiple products: a practical guide. Am J Manag Care. 2002;8:211-8.

32. Ben Amor Y, Day MS, Schluger NW. Preventing the next generation of extensively drug-resistant tuberculosis. Int J Tuberc Lung Dis. 2010;14:525-7.

33. Byun JH, Park JA, Kang HR, Shin JY, Lee EK. Comparison of effectiveness between delamanid and bedaquiline among patients with multidrugresistant tuberculosis: a Markov model simulation study. Clin Drug Investig. 2016;36(11):957-68.

34. Wolfson L, Wirth D, Gibbert J, Rutz S, Diel R. Costs and effectiveness of combination therapy with Bedaquiline and other anti-tuberculosis drugs in patients with multi- and extensively drug-resistant tuberculosis in Germany (PIN 62). 2014.

35. Goldhirsch A, Gelber RD, Simes RJ, Glasziou P, Coates AS. Costs and benefits of adjuvant therapy in breast cancer: a quality-adjusted survival analysis. J Clin Oncol. 1989;7:36-44.

36. clinicaltrials.gov. A Phase 3 Study Assessing the Safety and Efficacy of Bedaquiline Plus PA-824 Plus Linezolid in Subjects With Drug Resistant Pulmonary Tuberculosis. 2015. https://clinicaltrials.gov/ct2/show/ NCT02333799. Accessed 5 Dec 2016.

37. Nunn AJ, Rusen ID, Van DA, Torrea G, Phillips PP, Chiang CY, et al. Evaluation of a standardized treatment regimen of anti-tuberculosis drugs for patients with multi-drug-resistant tuberculosis (STREAM): study protocol for a randomized controlled trial. Trials. 2014;15:353.

38. Janssen. Press release: Janssen Collaborates for Continued Evaluation of Multidrug-Resistant Tuberculosis Treatment Regimens with SIRTURO ${ }^{\oplus}$ (bedaquiline). 2015. https://www.jnj.com/news/all/Janssen-Collaborates-forContinued-Evaluation-of-Multidrug-Resistant-Tuberculosis-TreatmentRegimens-with-SIRTURO-bedaquiline . Accessed 5 Dec 2016

39. Partners in Health (PIH), Médecins Sans Frontières (MSF), Interactive Research and Development (IRD). The endTB Project: Expanding New Drugs for TB. http://www.stoptb.org/wg/gli/assets/documents/M7/3.\%20RICH_ endTB\%20Project.pdf. Accessed 5 Dec 2016.

40. Guglielmetti L, Le DD, Jachym M, Henry B, Martin D, Caumes E, et al. Compassionate use of bedaquiline for the treatment of multidrug-resistant and extensively drug-resistant tuberculosis: interim analysis of a French cohort. Clin Infect Dis. 2015;60(2):188-94

41. Ndjeka N, Conradie F, Schnippel K, Hughes J, Bantubani N, Ferreira H, et al. Treatment of drug-resistant tuberculosis with bedaquiline in a high HIV prevalence setting: an interim cohort analysis. Int J Tuberc Lung Dis. 2015; 19:979-85.

42. Kuksa $L$. The role of bedaquiline for pre-XDR TB and XDR-TB treatment in Latvia: DR-TB Training Network webinar. 2015. https://drtbnetwork.org/sites/ default/files/The\%20role\%20of\%20Bedaquiline\%20for\%20preXDR\%20TB\%20Latvia.pdf. Accessed 5 Dec 2016.
43. Pym AS, Diacon AH, Tang SJ, Conradie F, Danilovits M, Chuchottaworn C, et al. Bedaquiline in the treatment of multidrug- and extensively drug-resistant tuberculosis. Eur Respir J. 2016;47(2):564-74.

44. Ndjeka N, Conradie F, Hughes J, Schnipel K, Cox H, Bantubani N, et al. Safe and effective bedaquiline treatment of drug-resistant tuberculosis (DR-TB) within the National Bedaquiline Clinical Access Programme in South Africa. In: The Union/CDC Late-breaker session on TB; 28 October, 2014. 2014.

45. Day M, Middlemiss A, Thorpe J, Okereke E. What really happens to tuberculosis patients classified as lost to follow-up in West Yorkshire? Euro Surveill. 2012;17(38).

46. Tang S, Yao L, Hao X, Zhang X, Liu G, Liu X, et al. Efficacy, safety and tolerability of linezolid for the treatment of XDR-TB: a study in China. Eur Respir J. 2015;45: $161-70$.

47. Lee M, Lee J, Carroll MW, Choi H, Min S, Song T, et al. Linezolid for treatment of chronic extensively drug-resistant tuberculosis. N Engl J Med. 2012;367: 1508-18.

48. Lee M, Cho SN, Barry III CE, Song T, Kim Y, Jeong I. Linezolid for XDR-TB-final study outcomes. N Engl J Med. 2015;373:290-1.

49. Jeong BH, Jeon K, Park HY, Kwon OJ, Lee KS, Kim HK, et al. Outcomes of pulmonary MDR-TB: impacts of fluoroquinolone resistance and linezolid treatment. J Antimicrob Chemother. 2015;70:3127-33.

50. Gupta R, Geiter LJ, Wells CD, Gao M, Cirule A, Xiao H. Delamanid for extensively drug-resistant tuberculosis. N Engl J Med. 2015;373:291-2.

51. Avong $Y K$, Isaakidis $P$, Hinderaker SG, Van den Bergh $R$, Ali $E$, Obembe $B O$, et al. Doing no harm? Adverse events in a nation-wide cohort of patients with multidrug-resistant tuberculosis in Nigeria. PLoS One. 2015;10:e0120161.

52. Lakhani N, Thompson W, Bombassaro AM. Probable linezolid-induced pancytopenia. Can J Infect Dis Med Microbiol. 2005;16:286-8.

\section{Submit your next manuscript to BioMed Central and we will help you at every step:}

- We accept pre-submission inquiries

- Our selector tool helps you to find the most relevant journal

- We provide round the clock customer support

- Convenient online submission

- Thorough peer review

- Inclusion in PubMed and all major indexing services

- Maximum visibility for your research

Submit your manuscript at www.biomedcentral.com/submit
Biomed Central 